Scientific Journal of Hamadan Nursing \& Midwifery Faculty - ISSN 2008-2819

\title{
The Effect of Kangaroo Mother Care Counseling on Postpartum Blues, A Randomized Clinical Trial
}

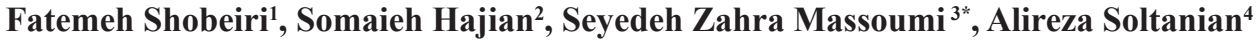 \\ 1. Professor of Midwifery, Mother and Child Care Center, Faculty of Nursing and Midwifery, Hamadan University \\ of Medical Sciences, Hamadan, Iran \\ 2. MSc in Midwifery Counseling, Students Research Committee, Faculty of Nursing and Midwifery, Hamadan \\ University of Medical Sciences, Hamadan, Iran \\ 3. Assistant Professor, Mother and Child Care Center, Hamadan University of Medical Sciences, Hamadan, Iran \\ 4. Associate Professor, Health Sciences Research Center, School of Public Health, Hamadan University of Medical \\ Sciences, Hamadan, Iran
}

\begin{tabular}{lll}
\hline Article Info & Abstract \\
\hline
\end{tabular}

Received: $\quad 2017 / 09 / 5$

Accepted: $\quad$ 2017/10/2

Published Online 2018/03/16

DOI: $10.30699 / \mathrm{sjhnmf.26.2.113}$

Original Article

Use your device to scan and read the article online

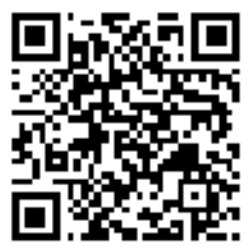

Introduction: Kangaroo mother care is a standard method based on skin contact with the mother and newborn skin. The aim of this study was to evaluate the effect of kangaroo mother care counseling on postpartum blues in women in Fatemieh Hospital, Hamadan.

Methods: A randomized clinical trial was conducted on 104 pregnant women who referred to Fatemieh hospital in Hamadan city, 2016. Those who had gestational age of 37-42 weeks were randomly divided in to two groups of case and control according to the day of delivery. Postpartum routine care was performed in both groups. In the case group in first 24 hours after delivery, kangaroo mother care was taken with a counseling session (20-30 minutes) was performed until the 10th day of delivery. The after birth Stein questionnaire was completed before and after intervention in two groups. Data was analyzed using SPSS16, t-test and ANOVA.

Results: The results showed that the intervention in the experimental group was about 4 times lower in postpartum distress score $(P<0.001)$. However, the control group also had a significant decrease on the 10th day before the intervention, but this decrease was higher in the experimental group $(P=0.009)$.

Conclusion: The results showed that kangaroo mother care could reduce the postpartum blues. Mother's kangaroo care after delivery is recommended to promote benefits for mother and baby.

Keywords: Postpartum Blues, Kangaroo Mother Care, Counseling

Copyright $($ 2018, Sci J Hamadan Nurs Midwifery Fac. This is an open-access article distributed under the terms of the Creative Commons Attribution-noncommercial 4.0 International License which permits copy and redistribute the material just in noncommercial usages, provided the original work is properly cited.

How to Cite This Article:

Shobeiri F, Hajian S, Massoumi Z, Soltanian A. The Effect of Kangaroo Mother Care Counseling on Postpartum Blues, A Randomized Clinical Trial. Sci J Hamadan Nurs Midwifery Fac. 2018; 26 (2): 113-119 


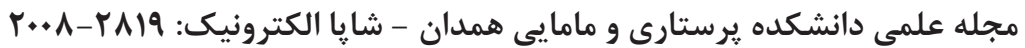

مقالأ يزوهشى همدان

تأثير مشاورة مراقبت آغوشى مادرانه بر اندوه يس از زايمان: يك كار آزمايى بالينى

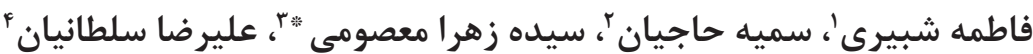

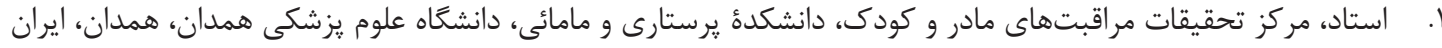

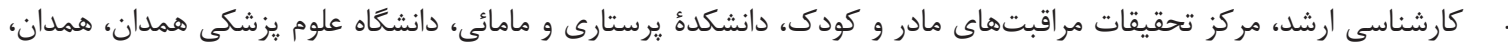

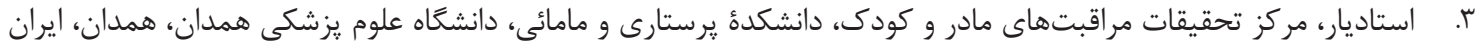

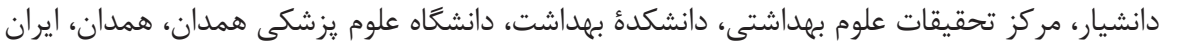

\begin{tabular}{|c|c|}
\hline جكيده & اطلاعات مقاله \\
\hline 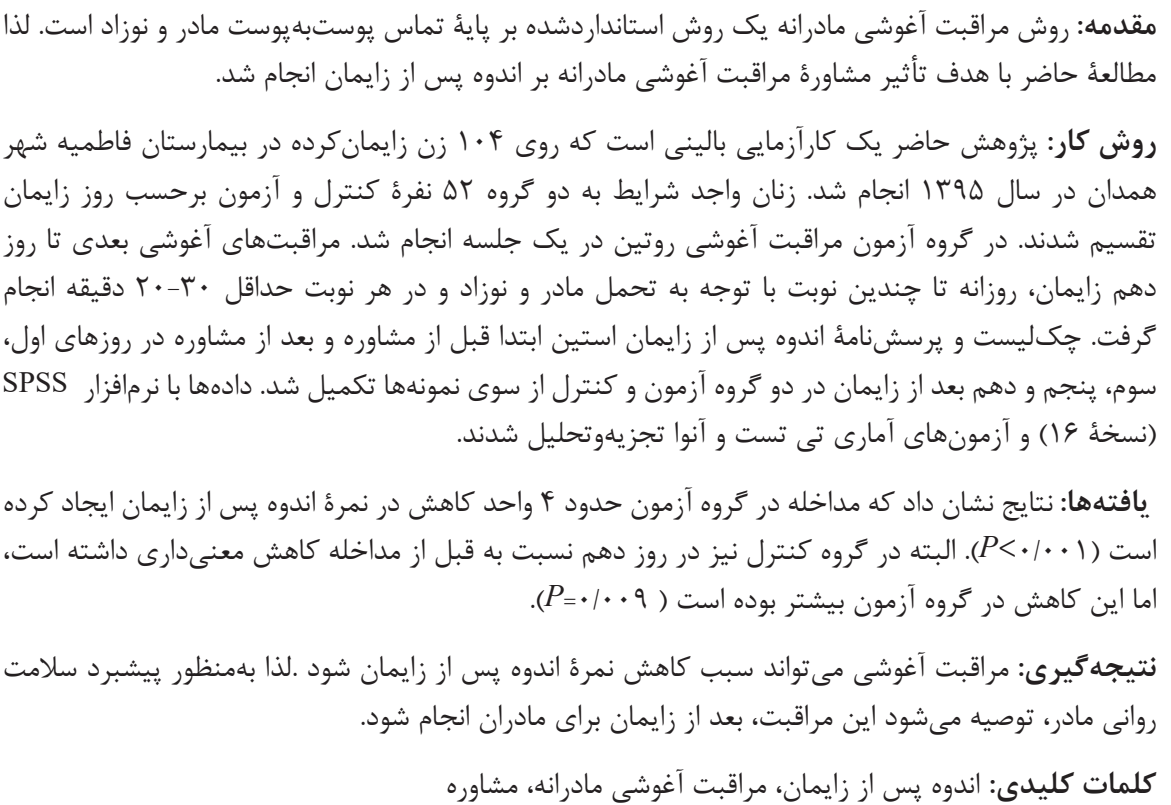 & 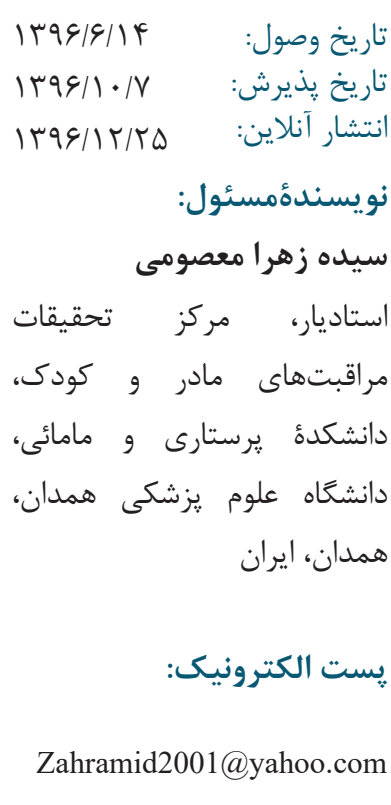 \\
\hline
\end{tabular}

مقلهمه

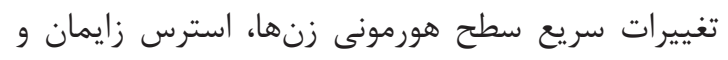

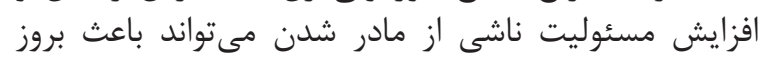

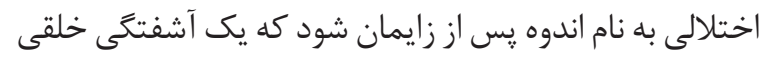

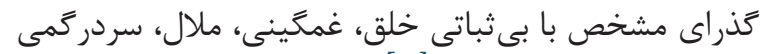

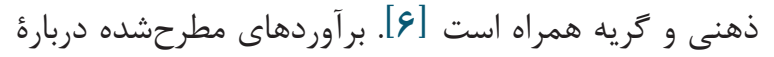

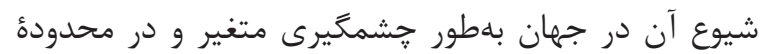

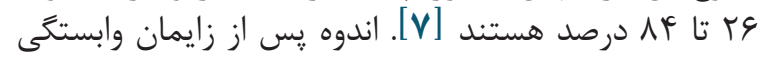

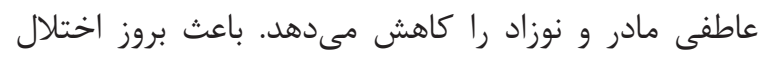

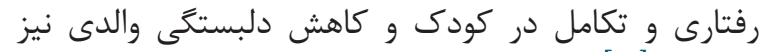

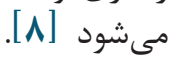

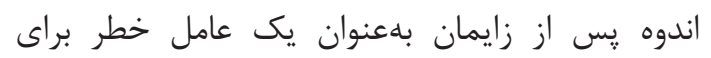

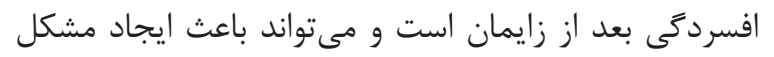

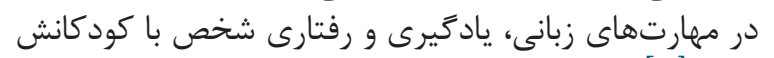

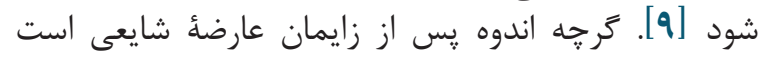

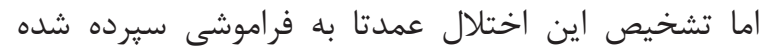

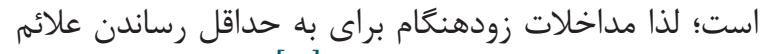

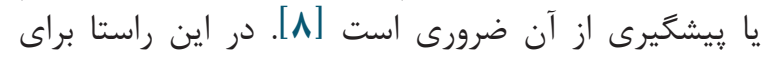

باردارى و زايمان مىتواند بر احساسات، رفتارها و تفكر

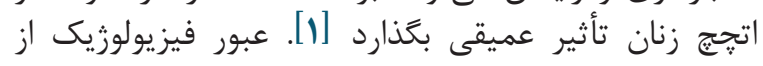

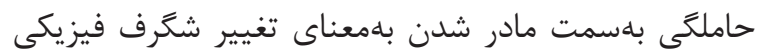

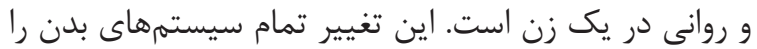

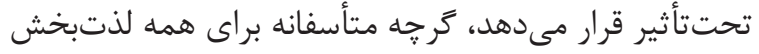

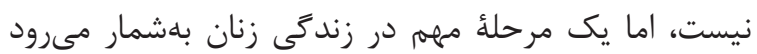

تولد نوزاد در بسيارى از زنان، باعث شروع تغيتيرات

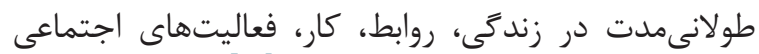

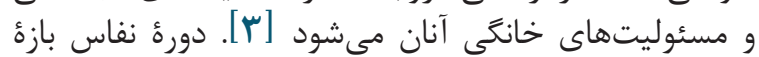

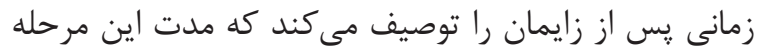

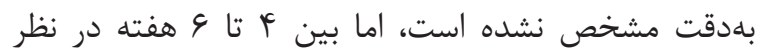

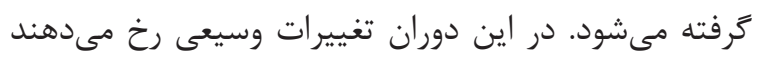

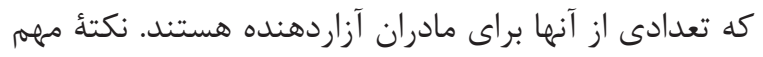

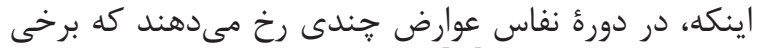

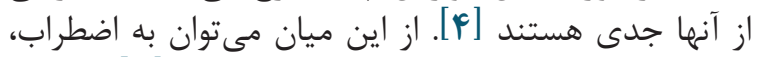

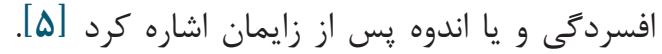


است كه 1 سؤال اول در طيف ه گزينهاى ليكرت از أ-

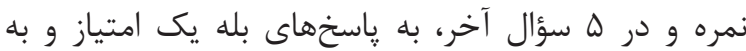

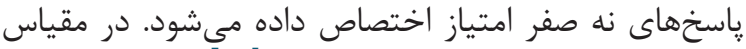

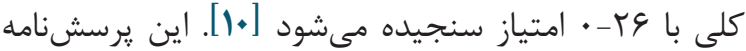

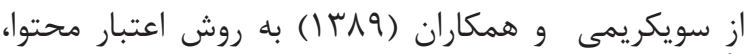

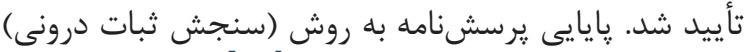

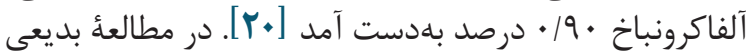

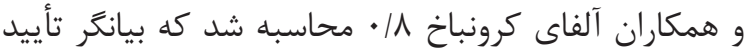

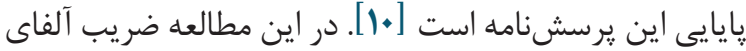

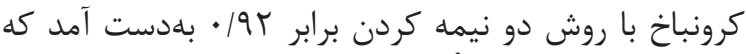

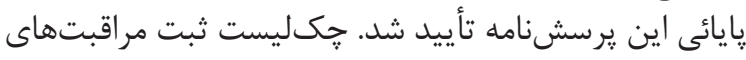

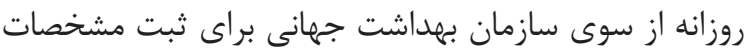

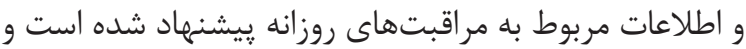

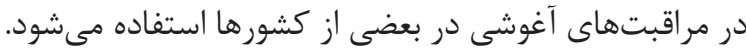

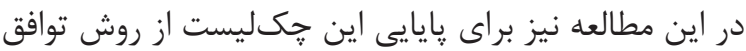

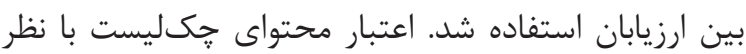

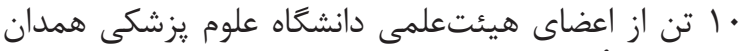

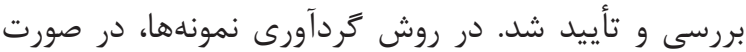

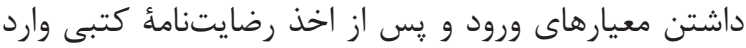

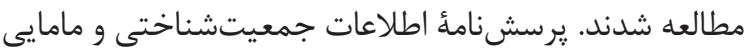

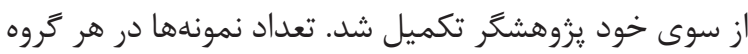

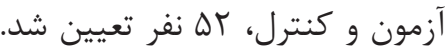

در زروه آزمون و حداكثر طى عاب ساعت اول يس از

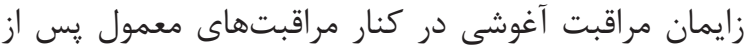

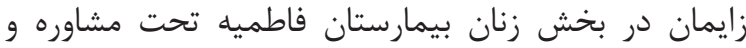

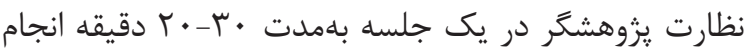

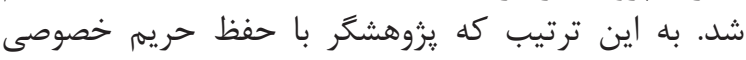

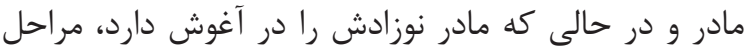

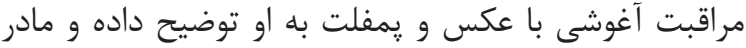

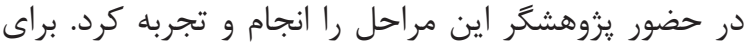

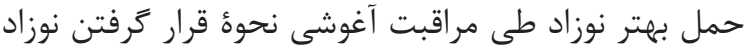

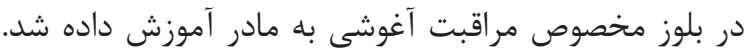

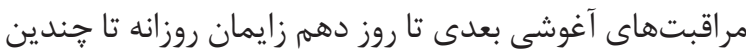

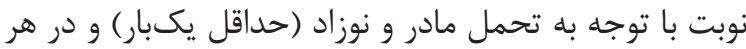

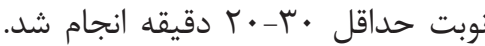

جك إنليست ثبت مراقبتهاى روزانه در اختيار مادران گروه

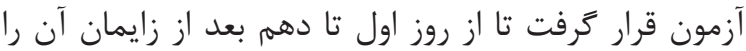

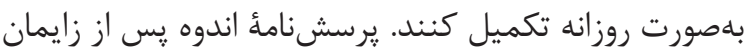

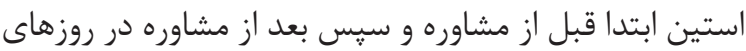

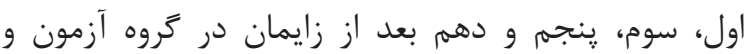

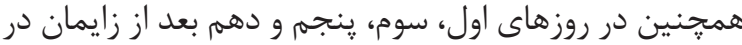

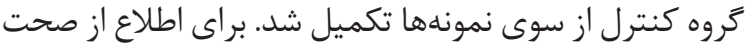

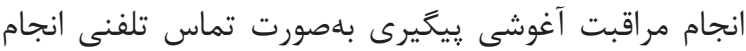

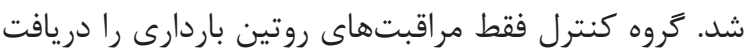

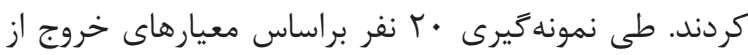

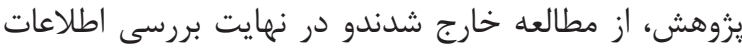

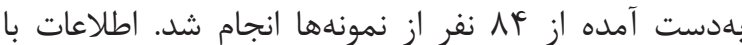

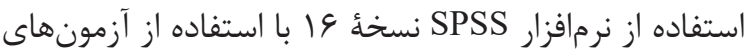
آمارى توصيفى و تحليلى (آزمون تى مستقل، ANOVA و
״يشخيرى و كاهش شدت اندوه پِ از زايمان اقداماتي

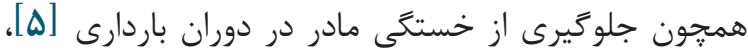

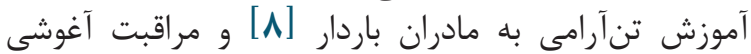

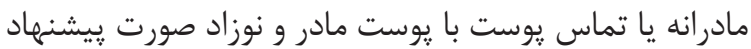

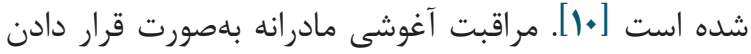

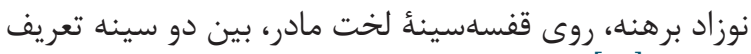

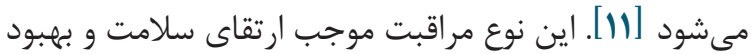

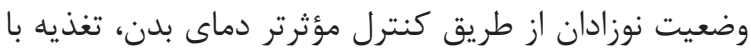

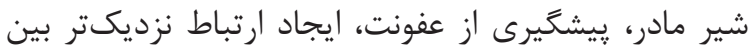

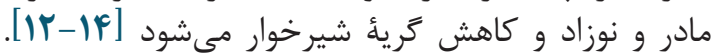
مراقبت آغوشى باعث ترشح اكسى توسين، افزايش آرامش،

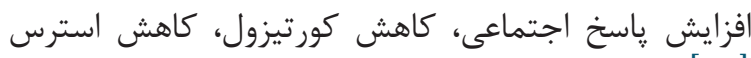

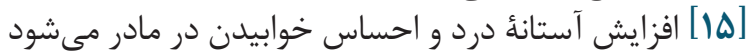

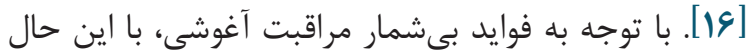

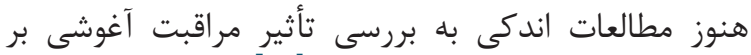

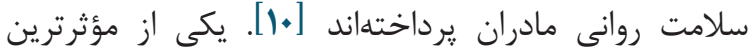

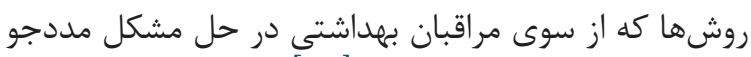

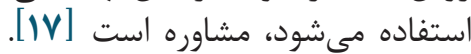

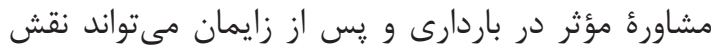

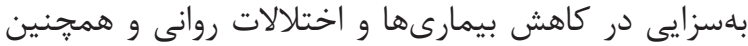

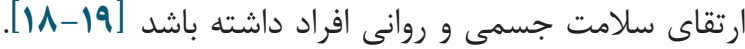

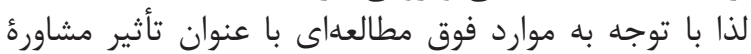

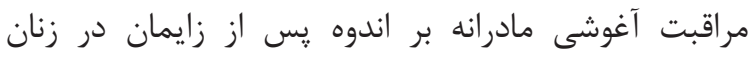

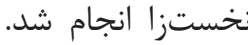

\section{روش كار}

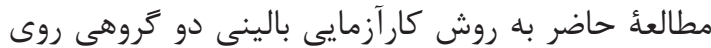

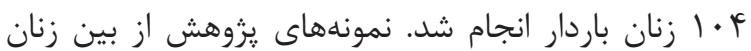

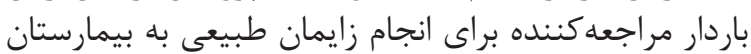

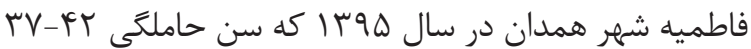

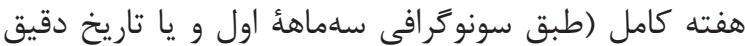

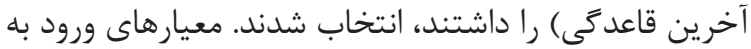

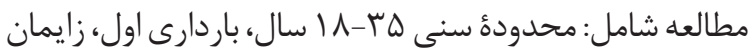

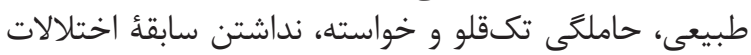

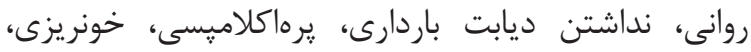

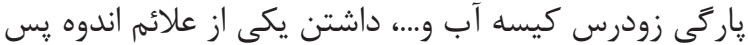

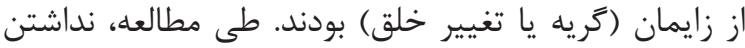

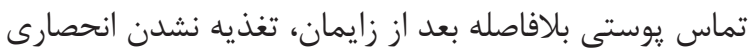

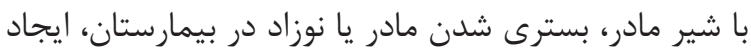

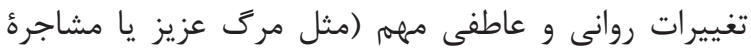

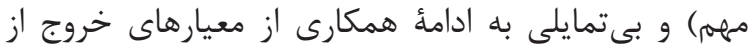

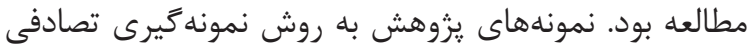

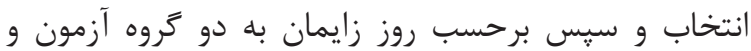

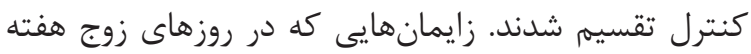

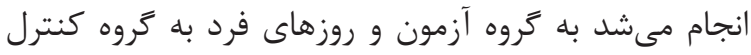

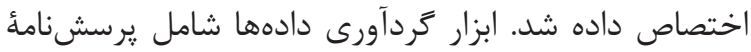

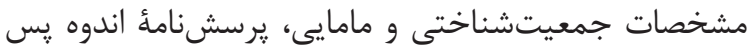

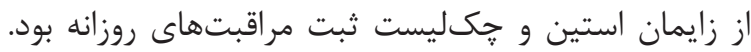

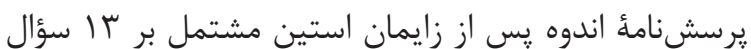




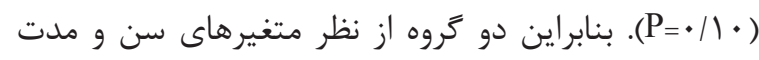
ازدواج همگَن بودند (جدول () ).

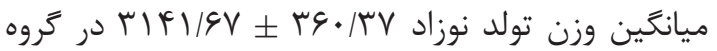

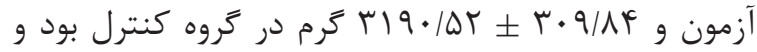

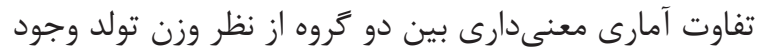

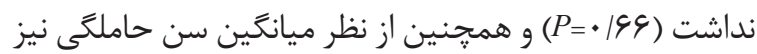

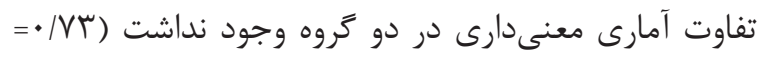

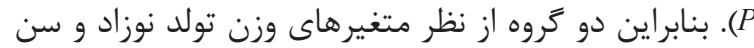
حاملگى همًًن بودند (جدول (1) ).

با توجه به نتايج جدول rا، تحليل دادههاى تكرارى نشان

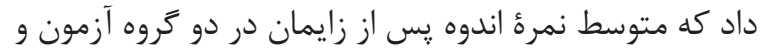

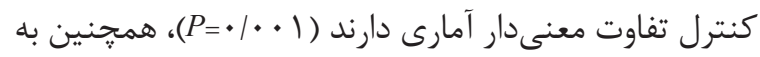
وضوح مشاهده مى شود كه متوسط نمرات اندوه يس از از زايمان

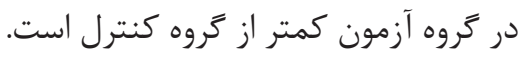

تحليل دادههاى تكرارى) تجزيهوتحليل شد. ميزان P كمتر

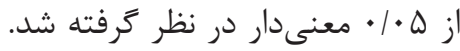

بافتهها.

در اين مطالعه f • ا زن نخستزا شركت كردند كه حين

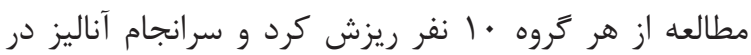

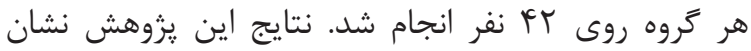

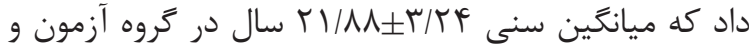

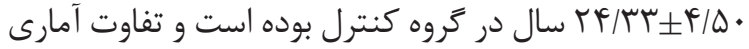

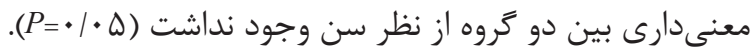

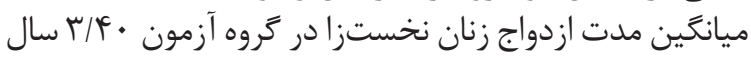

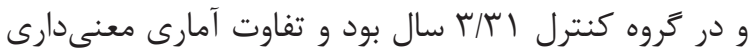

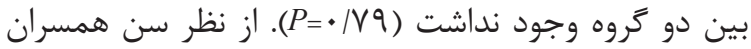

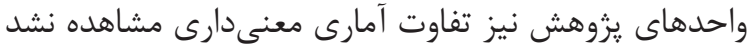

جدول ا. مقايسُٔ ميانغَين و انحر افمعيار برخى مشخصات دموتر افيك واحدهاى يزوهش در دو كروه آزمون و كنترل

\begin{tabular}{|c|c|c|c|}
\hline آزمون آمارى (تى- مستقل) & 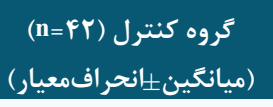 & 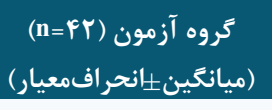 & متغيير \\
\hline$\cdot 1 \cdot \Delta$ & $r F / r r_{ \pm} F / \Delta$. & $r \mid / \Lambda_{ \pm} r / r F$ & سن (سال) \\
\hline$\cdot / v 9$ & $r / r \mid \pm 1 / 4 \Delta$ & $r / \mathcal{r} \cdot \pm 1 / \lambda r$ & مدت ازدواج (سال) \\
\hline .11 & $r \Lambda / q \cdot \pm F / l l$ & $r V / \varepsilon \cdot \pm r / \cdot q$ & سن همسر (سال) \\
\hline .199 & $r 19 \cdot / \Delta r \pm r \cdot q / \Delta F$ & $r|F| / q V_{ \pm} r q \cdot / r V$ & وزن تولد (كرم) \\
\hline 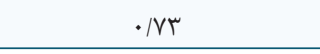 & $r q / \cdot \Delta_{ \pm} 1 / \cdot v$ & rq// $r_{ \pm} \cdot / 99$ & سن حاملكَى (هفته) \\
\hline
\end{tabular}

جدول r. مقايسهُ ميانگين و انحرافمعيار نمرهٔ اندوه يُ از زايمان در دو كروه آزمون و كنترل با استفاده از تحليل دادههاى تكرارى

تروهها:

\begin{tabular}{|c|c|c|c|c|c|c|c|}
\hline$F=1 \cdot / 9 \Delta r$ & $F=F / T)$ & $1 / r \pm r / r q$ & $r / F T \pm T / T G$ & $r / r \Delta \pm f / r f$ & $\Delta / \cdot r \pm r / 9 \Lambda$ & $9 / 9 T \pm r / T F$ & آزمون \\
\hline$P=\cdot / . .1$ & $P=\cdot / \cdot \Delta T$ & & & & & & \\
\hline & & $r / l \uparrow \pm r / \Delta r$ & $\varepsilon / F V \pm F / F \Delta$ & $\varepsilon / Q \Delta \pm r / r \digamma$ & $\Delta / q \pm r / \uparrow \Lambda$ & $\Delta / q \pm r / F \Lambda$ & كنترل \\
\hline
\end{tabular}

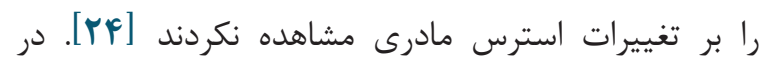

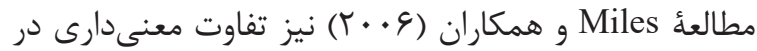

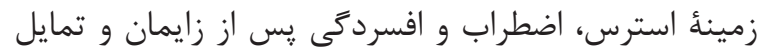

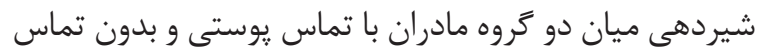

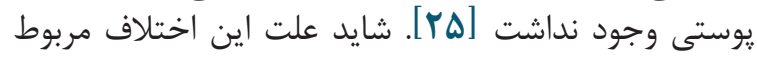

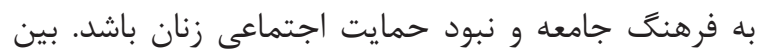

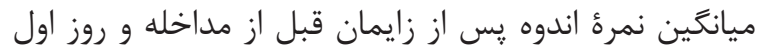

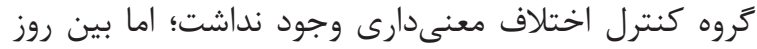

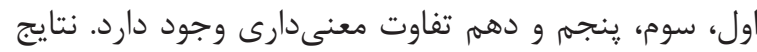

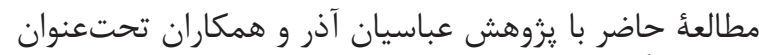

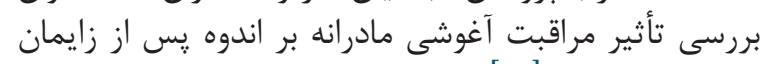

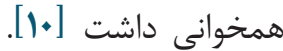

مطالعه نشان داد كه ميانخين نمره اندوه يس از زايمان

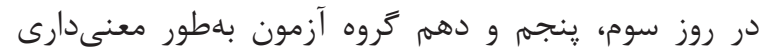

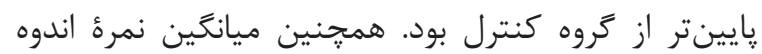

\section{بحث}

در اين مطالعه ميانگين نمره اندوه در كروه آزمون كمتر

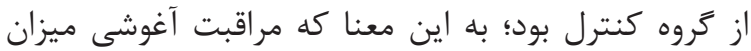

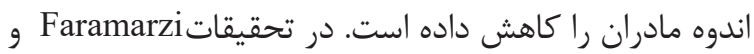

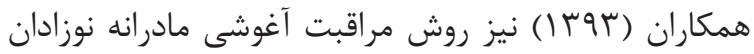

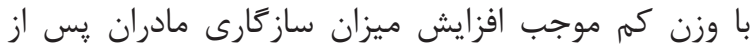

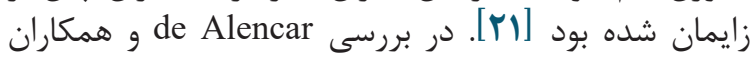

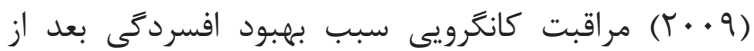

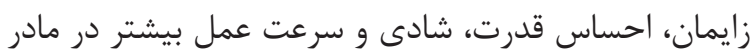

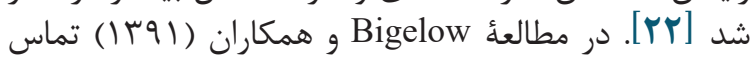

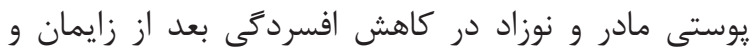

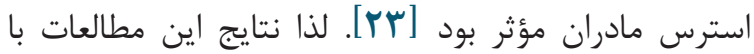

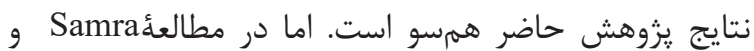

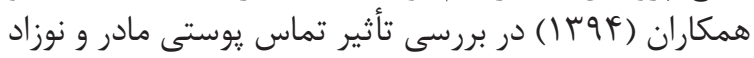

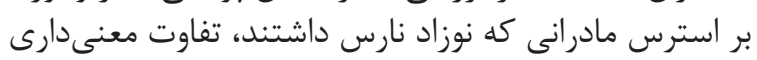


جهارم پֶ از زايمان عنوان كرده بود [1]. علت دقيق اندوه پِ إز زايمان شناخته نشده است،

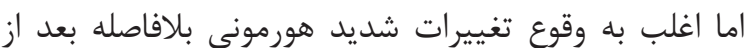

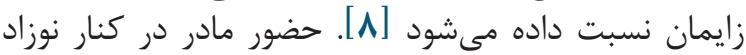

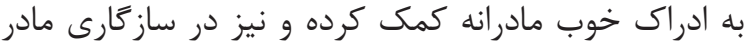

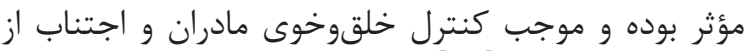

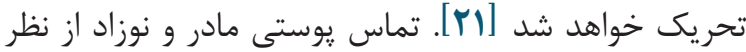

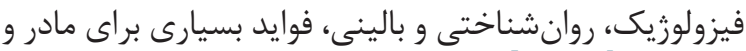

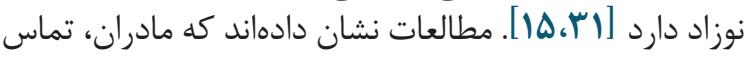

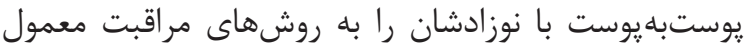

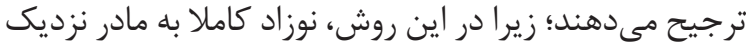

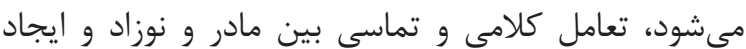

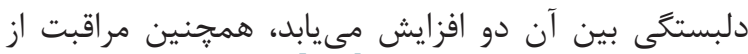

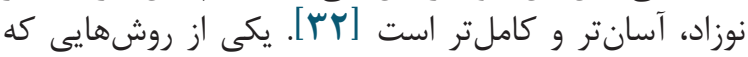

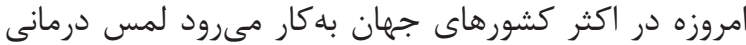

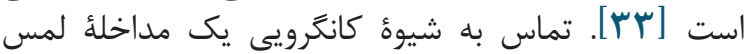

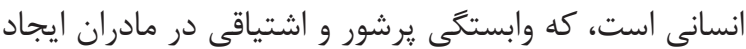

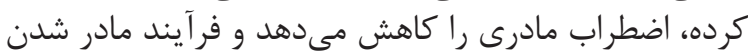

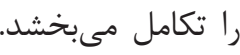

احتمال از دست دادن نمونهها در طول مدت إِيخيرى

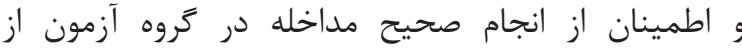
محدوديتهاى اين يزوهش إن بوده است.

\section{نتيجه تيرى}

نتايج حاصل از اين مطالعه بيانكر آن است كه مشاورة

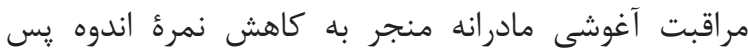

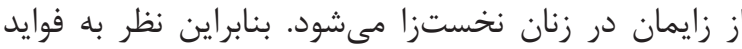

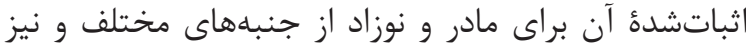

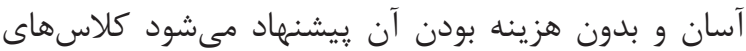

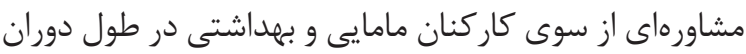

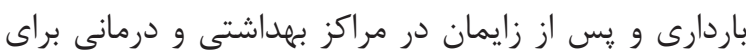

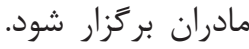

\section{سياسخز ارى}

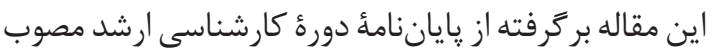
دانشخاه علوم يزشكى همدان با كد اخلاق . UMSHA.REC يروهشى دانشكاه علوم يزشكى همدان كه امكان انجان انجام اين

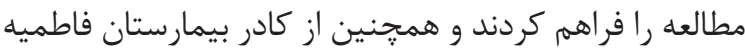

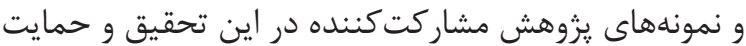

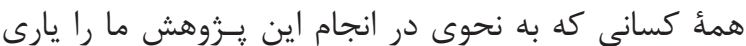

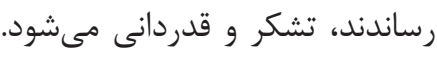

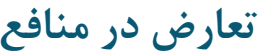

هيجَّونه تضاد منافعى وجود ندارد.

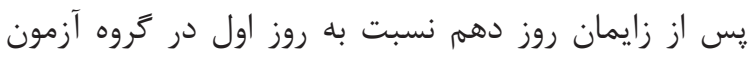

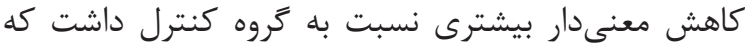

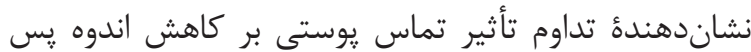

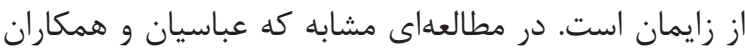

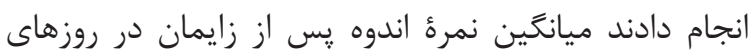

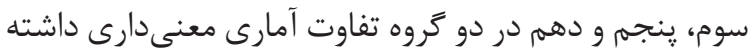

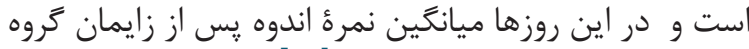

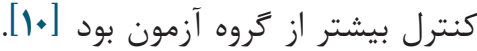

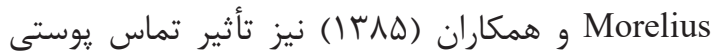

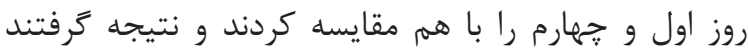

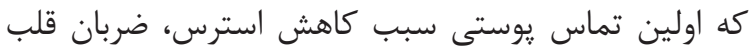

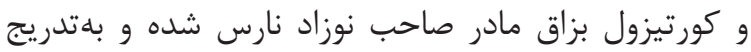

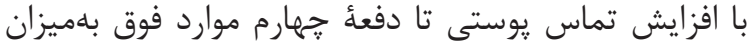

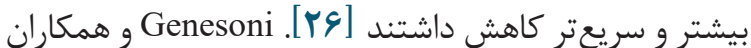

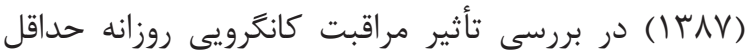

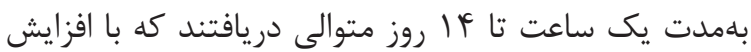

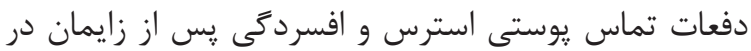

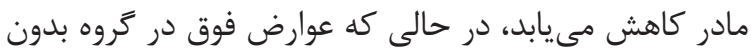

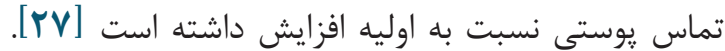

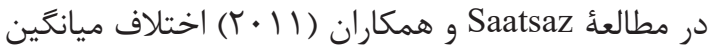

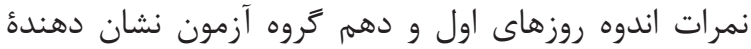

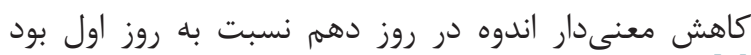

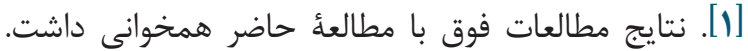

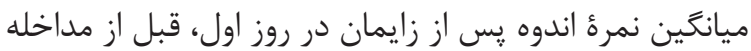

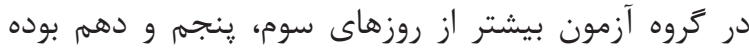

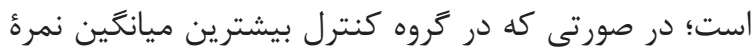

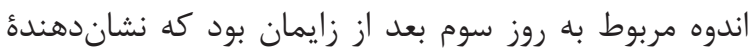

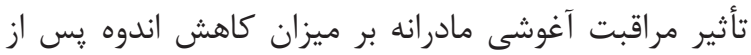

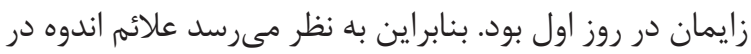

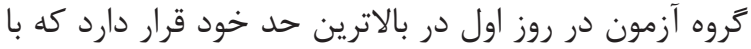

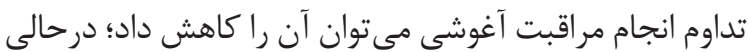

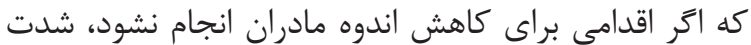

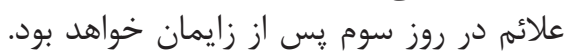
Adewuya

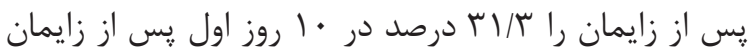

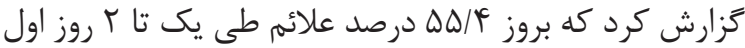

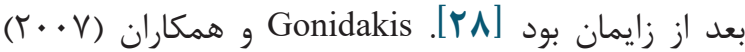

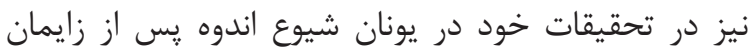

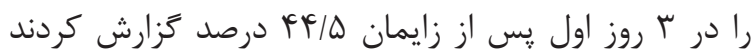

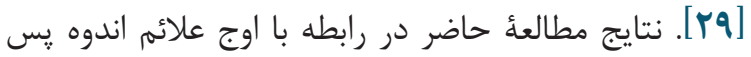

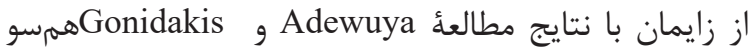
بود. در مطالعئ Nematbakhsh و همكاران بر زنان سران سزارينى

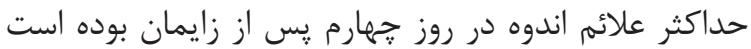

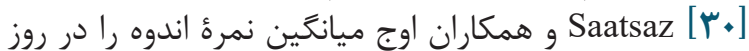




\section{References}

1. Saatsaz S, Rezaei R, Sharifnia H, Kheirkhah F, Moulookzadeh S, Haji Hosseini F. Effect of mother and newborn skin to skin contact on postpartum blues. J Babol Univ Med Sci. 2011;13(3):5965. http://jbums.org/article-1-3804-en.html

2. Soltani F, Shobeiri F. Mensatrual patterns and its disorders in high school girls. The Iraian $\mathrm{J}$ of Obstetrics , Gynecology \& Infertility. 2011;14(1):28-36. http://www.sid.ir/En/Journal/ ViewPaper.aspx?ID=205739

3. Akbarzadeh M, Shobeyri F, Mahjub H, Ebrahimi R. Investigating the factors influencing the duration of beginning delivery to hospital discharge using cox regression model. The Iranian Journal of Obstetrics, Gynecology \& Infertility. 2014;17(105):1-9. http://ijogi.mums.ac.ir/article_3056.html

4. Cunningham G, Leveno K, Bloom S, Spong C, Dashe J, Hoffman B, et al. Williams obstetrics. 24nd ed. New York: McGraw-Hill; 2014. p.797.

5. Banafsheh E, Salari P, Hebrani P, Dadgar S, Rahmani R, Bahri N. Relationship between maternal fatigues during pregnancy with postpartum blues. The Iranian Journal of Obstetrics, Gynecology and Infertility. 2013;16(54):7-15. http://www. sid.ir/En/Journal/ViewPaper.aspx?ID=197138

6. Sadock B, sadock V, Ruiz P. Kaplan \& Sadock's comprehensive textbook of psychiatry. 11nd ed. Philadelphia: Lippincott Williams \& Wilkins; 2015. p.181.

7. Danforth DN, Gibbs RS. Danforth's obstetrics and gynecology. 10nd ed. Philadelphia: Lippincott Williams \& Wilkins; 2008. p.623-4.

8. Akbarzadeh M, Toosi M, Zare N, Sharif F. Effect of Relaxation Training to Pregnant Mothers on Quality of life and Postpartum Blues. Knowledge Health. 2012;7(2):83-8. http://knh.shmu.ac.ir/index.php/site/article/view/95

9. Im Sj, Shin $\mathrm{Cj}$. The effects of music on the frontal EEG asymmetry of the mothers with postpartum blues. Korean J Biol Sci. 2011;18(3):134-40.

10. Badiee Z, Faramarzi S, MiriZadeh T. The effect of kangaroo mother care on mental health of mothers with low birth weight infants. Adv Biomed Res. 2014;20(3):214. https://doi.org/10.4103/2277-9175.143262 PMID:25371871 PMCID:PMC4219210

11. Frederick AC, Busen NH, Engebretson JC, Hurst NM, Schneider KM. Exploring the skin to skin contact experience during cesarean section. J Am Assoc Nurse Pract. 2015;28(1):31-38. https://doi. org/10.1002/2327-6924.12229 PMID:25764023

12. Arzani A, Zahedpasha Y, Ahmadpour M, Khafri S, Khaikhah F, Aziznejad P. Kangaroo care effect on self-esteem in the mothers on low birth weight infants. Journal Babol university medical sicences. 2012; 14(3):52-58.

13. de Alba-Romero C, Camaño-Gutiérrez I, López-Hernández $\mathrm{P}$, de Castro-Fernández J, Barbero-Casado $\mathrm{P}$, Salcedo-Vázquez $\mathrm{ML}$, et al. Postcesarean section skin to skin contact of mother and child. J Hum Lact. 2014;30(3):2836. https://doi.org/10.1177/0890334414535506 PMID:24847031

14. Olsson E, Ahlsen G, Eriksson M. Skin-to-skin contact reduces near-infrared spectroscopy pain responses in premature infants during blood sampling. Acta Paediatr. 2015;105(4):376-80. https:// doi.org/10.1111/apa.13180 PMID:26342142

15. Moore ER, Anderson GC, Bergman N, Dowswell T. Early skin-to-skin contact for mothers and their healthy newborn infants. Cochrane Database Syst Rev. 2012;5:CD003519. https:// doi.org/10.1002/14651858.CD003519.pub3 PMID:22592691 PMCID:PMC3979156

16. Nahidi F, Dorii F, Ravari M, Akbarzadeh Baghban A. Effect of mother and newborn skin to skin contact on rate mothers satisfaction. Shahih Beheshti Univ Med Scie Health Serv. 2010;20(71):1-5.

17. Raisi Dehkordi Z, Raei M, Ghassab Shirazi M, Raisi Dehkordi SAR, Mirmohammadali M. Effect of Telephone Counseling on Continuity and Duration of Breastfeeding among Primiparus Women. Hayat. 2012;18(2):57-65. http://hayat. tums.ac.ir/article-1-31-en.html

18. Akbarzade M, Toosi M, Zare N, Sharif F. Effect of Relaxation and Attachment Behaviors Training on Anxiety in First-time Mothers in Shiraz City, 2010: A Randomized Clinical Trial. Qum univ Med sci J. 2012;6(4):14-23. http://journal. muq.ac.ir/article-1-125-en.html

19. Azizi M, Lamyian M, Faghihzade S, Nematollazade M. The effect of counseling on anxiety after traumatic childbirth in nulliparous women; a single blind randomized clinical trial. J Kermanshah Univ Med Sci. 2010; 14(3):219-27.

20. Karimi S, Kariman N, Shams J, Nassir A, Aliakbari S. Relationship between postpartum blues with anxiety and postpartum depression in Tehran women. Thesis for M.Sc degree in midwifery. 2010.

21. Faramarzi S, Badiee Z, Miri T, Khodadad Z. The effectiveness of kangaroo mother's care (KMC) method of low birth weight infants on maternal adjustment. Iran J Nurs Res. 2014;8(4):29-41. http://ijnr.ir/article-1-1304-en.html

22. De Alencar AE, Arraes LC, De Albuquerque EC, Alves JG. Effect of kangaroo mother 
care on postpartum depression. J Trop Pediatr. 2009;55(1):36-8. https://doi.org/10.1093/tropej/ fmn083 PMID:19066171

23. Bigelow A, Power M, MacLellan-Peters J, Alex M, McDonald C. Effect of Mother/Infant Skin-to-Skin Contact on Postpartum Depressive Symptoms and Maternal Physiological Stress. J Obstet Gynecol Neonatal Nurs. 2012;41(3):369-8. https://doi.org/10.1111/ j.1552-6909.2012.01350.x PMID:22537390

24. Samra HA, Dutcher J, McGrath JM, Foster M, Klein L, Djira G, et al. Effect of Skin-to-Skin Holding on Stress in Mothers of Late-Preterm Infants: A Randomized Controlled Trial. Adv Neonatal Care. 2015;15(5):354-64. https:// doi.org/10.1097/ANC.0000000000000223 PMID:26356086

25. Miles R, Cowan F, Glover V, Stevenson J, Modi N. A controlled trial of skin to skin contact in extremely preterm infants. Early Hum Dev. 2006;82(7):447-55. https://doi.org/10.1016/j. earlhumdev.2005.11.008 PMID:16458458

26. Morelius E, Theodorsson E, Nelson N. Salivary cortisol and mood and pain profiles during skin to skin care for an unselected group of mothers and infants in neonatal intensive care. Pediatrics. 2005;116(5):1105-13. https://doi.org/10.1542/ peds.2004-2440 PMID:16263996

27. Genesoni L, Curran RL, Huertas-Ceballos A, Tallandini MA. Kangaroo mother care and its effect on parenting stress and maternal postnatal attachment in case of premature birth. Arch Dis Child Fetal Neonatal Ed. 2008;93:80.

28. Adewuya AO. The maternity blues in West- ern Nigerian women: prevalence and risk factors. Am J Obstet Gynecol. 2005;193(4):15225. https://doi.org/10.1016/j.ajog.2005.02.085 PMID: 16202750

29. Gonidakis F, Rabavilas AD, Varsou E, Kreatsas G, Christodoulou GN. Maternity blues in Athens, Greece: A study during the first 3 days after delivery. J Affect Disord. 2007;99(1-3)10715. https://doi.org/10.1016/j.jad.2006.08.028 PMID: 17011042

30. Nematbakhsh F, Kordi M, Sahebi A, Esmaeeli H. The effect of skin to skin contact on the severity of postpartum blues of cesarean sectioned women. J Fundam Ment Health. 2004;6(23-24):89-98.

31. Curley JP, Jensen CL, Franks B, Champagne FA. Variation in maternal and anxiety-like behavior associated with discrete patterns of oxytocin and vasopressin 1a receptor density in the lateral septum. Horm Behav. 2012;61(3):45461. https://doi.org/10.1016/j.yhbeh.2012.01.013 PMID:22300676 PMCID:PMC3312967

32. Velandia M, Matthisen AS, Uvnäs-Moberg K, Nissen E. Onset of vocal interaction between parents and newborns in skin-to-skin contact immediately after elective cesarean section. Birth. 2010;37(3):192-201. https:// doi.org/10.1111/j.1523-536X.2010.00406.x PMID:20887535

33. de Macedo E C, Cruvinel F, Lukasova K, D Antino MEF. The mood variation in mothers of preterm infants in kangaroo mother care and conventional incubator care. J Trop Pediatr. 2007;53(5):3446. https://doi.org/10.1093/tropej/fmm076 PMID:17881409 